\title{
The Impacts of Synbiotic Supplementation on
} Periodontal Indices and Biomarkers of Oxidative

\section{Stress in Type 2 Diabetes Mellitus Patients with Chronic Periodontitis Under Non-Surgical Periodontal Therapy. A Double-Blind, Placebo-Controlled Trial}

This article was published in the following Dove Press journal:

Diabetes, Metabolic Syndrome and Obesity: Targets and Therapy

\author{
Hadi Bazyar (iD) ${ }^{1,2}$ \\ Leila Maghsoumi-Norouzabad (iD) ${ }^{3}$ \\ Mohsen Yarahmadi ${ }^{3}$ \\ Hassan Gholinezhad ${ }^{3}$ \\ Leila Moradi ${ }^{4}$ \\ Parvin Salehi ${ }^{5}$ \\ Mohammad Hosein \\ Haghighi-zadeh ${ }^{6}$ \\ Ahmad Zare Javid ${ }^{1,3}$ \\ 'Nutrition and Metabolic Diseases \\ Research Center, Ahvaz Jundishapur \\ University of Medical Sciences, Ahvaz, \\ Iran; ${ }^{2}$ Student Research Committee, \\ Ahvaz Jundishapur University of Medical \\ Sciences, Ahvaz, Iran; ${ }^{3}$ Department of \\ Nutrition, School of Allied Medical \\ Sciences, Ahvaz Jundishapur University of \\ Medical Sciences, Ahvaz, Iran; ${ }^{4}$ Health \\ Research Institute, Diabetes Research \\ Center, Ahvaz Jundishapur University of \\ Medical Sciences, Ahvaz, Iran; \\ ${ }^{5}$ Department of Periodontology, School \\ of Dentistry, Ahvaz Jundishapur \\ University of Medical Sciences, Ahvaz, \\ Iran; ${ }^{6}$ Department of Biostatistics, School \\ of Public Health, Ahvaz Jundishapur \\ University of Medical Sciences, Ahvaz, \\ Iran
}

Correspondence: Ahmad Zare Javid Nutrition and Metabolic Diseases Research Center, Ahvaz Jundishapur University of Medical Sciences, Ahvaz, Iran

Tel +98914317 6237

Email ahmaddjavid@gmail.com
Aim: The aim of the current study was to investigate that combination of the synbiotic supplementation in adjunct with non-surgical periodontal therapy (NSPT) is useful in treating periodontitis and biomarkers of oxidative stress in type 2 diabetes mellitus (T2DM) patients. Methods: In this study, 47 patients suffering from DM and CP were recruited and randomly assigned to two groups. The intervention $(n=23)$ and control $(n=24)$ groups received either multispecies probiotic supplement plus $100 \mathrm{mg}$ fructo-oligosaccharide $(500 \mathrm{mg}$ in each capsule) or placebo capsule containing $500 \mathrm{mg}$ wheat flour, respectively, every day for 8 weeks. All subjects were treated with NSPT during the intervention period. Serum levels of interleukin-1 $\beta$ (IL-1 $\beta$ ), malondialdehyde (MDA), total antioxidant capacity (TAC), superoxide dismutase (SOD), catalase (CAT), glutathione peroxidase (GPx) and periodontal indices including clinical attachment loss (CAL), pocket depth (PD) (main outcome), BOP, and plaque index were measured before and after the intervention.

Results: Supplementation with the synbiotic with NSPT led to a significant decrease in the levels of IL-1 $\beta$, MDA, plaque index, PD, and CAL in the intervention group post-intervention $(\mathrm{p}<0.05)$. The serum levels of TAC, SOD, and GPx were significantly increased in the intervention group compared with the baseline $(\mathrm{p}<0.05)$. Consumption of synbiotic with NSPT, compared to the control, resulted in a significant decrease in the mean changes of IL-1 $\beta$, MDA, and CAL ( $p<0.05)$. Also, the mean changes of TAC and GPx were significantly higher in the intervention group compared with the control group $(p<0.05)$. Also, in the intervention group was seen a significant reduction in plaque index $(\mathrm{p}=0.035)$ and $\mathrm{BOP}(\mathrm{p}=0.04)$ compared with the control group.

Conclusion: It was observed that synbiotic supplementation with NSPT may be beneficial in improving inflammatory, antioxidant, and periodontal status in T2DM patients with CP.

Keywords: type 2 diabetes mellitus, inflammation, antioxidant, periodontal disease, synbiotic, non-surgical periodontal therapy

\section{Introduction}

Periodontal disease and type 2 diabetes mellitus (T2DM) hold a physiological birelationship with greater destruction of periodontal tissue and poorer glycemic 
control in diabetic patients with periodontal disease. ${ }^{1}$ Actually, in both periodontal diseases and DM, the major inflammatory markers with both systemic (impaired glycemic control) and local (periodontal destruction) effects are involved. ${ }^{2,3}$ It is shown that in T2DM patients with periodontal disease the levels of inflammatory markers such as hs-CRP, IL- $1 \beta$, TNF- $\alpha$, and IL- 6 are higher than diabetic patients without periodontal, which may adversely affect blood glucose and lipid metabolism. ${ }^{4}$ Also, the imbalance between the production of reactive oxygen species (ROS) and antioxidant defenses leading to oxidative stress is considered as an important factor involved in the development of DM and periodontal disease. ${ }^{5}$ According to some studies, the lipid peroxidation, MDA levels, and oxidative stress were increased in patients with periodontal disease $^{6}$ and DM. ${ }^{7}$ The findings of Thomas et al study indicated that serum levels of TAC were higher in the systemically healthy group without periodontitis and lower in the systemically healthy with chronic periodontitis (CP). In addition, serum SOD levels were highest in the DM-CP group because of a protective and adaptive mechanism against the oxidative stress developed in the tissue. $^{8}$ Therefore, the successful management of periodontal infection in diabetic patients may reduce local signs and symptoms and lead to better control of non-insulin dependent DM and metabolism. ${ }^{9}$

Studies have shown that NSPT including oral health education, scaling, and root planning have beneficial effects on periodontal disease management. ${ }^{10}$ Furthermore, several studies showed the useful effects of prebiotics, probiotics, and synbiotics on oxidative stress. ${ }^{11,12}$ Recently, using probiotics in order to improve metabolic profile, ${ }^{13}$ periodontal status, ${ }^{14}$ inflammatory factors, ${ }^{15}$ and biomarkers of oxidative stress ${ }^{16,17}$ has received great attention. A clinical trial study showed the use of probiotics could reduce periodontitis and inflammation in T2DM. ${ }^{18}$ Another study found effective periodontal therapy improved periodontal health status and insulin sensitivity and reduced insulin resistance in patients with T2DM and chronic periodontitis. ${ }^{19}$ Studies have shown that probiotics can influence metabolic profile through several mechanisms including enzymatic deconjugation of bile acids, conversion of cholesterol into coprostanol in the gut, ${ }^{20}$ and improving insulin sensitivity. ${ }^{21}$ Probiotics also can scavenge superoxide and hydroxyl radicals, ${ }^{22}$ increase glutathione (GSH) levels, ${ }^{23}$ decrease serum levels of IL-1 $\beta$, TNF- $\alpha$, and $\mathrm{CRP}^{24}$ and decrease expression of IL- 6 in adipocyte, and decrease adiposity, ${ }^{25}$ which may result in decreasing inflammation and oxidative stress. Several studies have investigated the effects of NSPT on the glycemic control of diabetic patients. Both non-diabetic and diabetic patients showed similar short-term outcomes after NSPT in terms of reducing probing depth and CAL and changes in subgingival microbiota. ${ }^{26}$ In addition, the results of a clinical study showed that NSPT was associated with a significant decrease in serum levels of TNF- $\alpha$ and IL-17 in patients with periodontal disease. ${ }^{27}$ According to the above effects, the hypothesis of this study was that the use of synbiotic with NSPT is effective in lowering the indicators of the presence of periodontitis and biomarkers of oxidative stress against the lack of effect. Since there were no studies to investigate the effects of multispecies synbiotic supplement in adjunct with NSPT in T2D patients with periodontal disease, the aim of the current study was to investigate that combination of the synbiotic supplementation in adjunct with NSPT is useful in treating periodontitis and biomarkers of oxidative stress in T2DM patients.

\section{Materials and Methods Subjects and Study Design}

This clinical randomized, parallel intervention study conformed to the ethical guidelines of the 1975 Declaration of Helsinki and was approved by the Ethics Committee of Ahvaz Jundishapur University of Medical Sciences (Ethical Code: IR.AJUMS.REC.1396.578 and registration code of Iran clinical trials: IRCT20161104030694N2). Of the 90 patients, 40 patients were excluded from the study (due to lack of inclusion criteria $(n=23)$ and disapproval to participate in the study $(n=17)$ and 50 patients were selected to participate in the study.

In this double-blind (participants and investigator) randomized clinical trial 50 patients (30-60 years old) with T2DM with periodontal diseases were selected from the Endocrinology clinic of Golestan Hospital in Ahvaz city, Iran, Ahvaz Jundishapur University of Medical Sciences. The sample size was determined based on PD (Pocket depth) (1.31 \pm 0.49 vs $0.49 \pm 0.39$, respectively, reduction in the intervention and control group) as the main outcome obtained from the study by Vivekananda et $\mathrm{al}^{14}$ For $\alpha$ value equal to 0.05 (confidence level of 95\%) and a power of $90 \%$, the sample size was computed using this formula: $n=\frac{\left(z_{1}-\frac{\alpha}{2}+z_{1}-\beta\right)^{2}\left(\delta_{1}{ }^{2}+\delta_{2}{ }^{2}\right)}{\left(\mu_{1}-\mu_{2}\right)^{2}}(\alpha=0.05$ and $\beta=$ $0.1)$ as 22 subjects per group. Considering the withdrawal rate of 15\%, 25 T2DM patients with chronic periodontal disease were selected for each group. According to the guideline, subjects who had FBG $\geq 126 \mathrm{mg} / \mathrm{dL}$ or $2 \mathrm{hr}$ 
glucose $(2 \mathrm{hpp}) \geq 200 \mathrm{mg} / \mathrm{dL}$ or $\mathrm{HbA1c} \geq 6.5 \%$ were diagnosed as diabetes mellitus. ${ }^{28}$ The inclusion criteria included: male or female aged between 30 and 60 years old; confirmed diabetes mellitus (no more than 5 years since diagnosis); mild and moderate periodontal disease that diagnosed by a periodontologist based on a pocket depth $\geq 4 \mathrm{~mm}$ and CAL $=1-4 \mathrm{~mm}$ in at least one site in three quarters of the mouth and radiographic photos ${ }^{29}$ and body mass index (BMI) ranged between 18.5 and $30 \mathrm{~kg} /$ $\mathrm{m}^{2}$. Subjects were excluded if they had the following criteria: hospitalized due to any complications of diabetes, any diseases affect levels of glycosylated hemoglobin such as anemia, hemodialysis, hemoglobinopathies, uremia, pregnancy and lactation, travel more than 2 weeks, smoking, other serious systemic diseases, noticeable change in diet in the past 6 months, using antibiotics, noticeable change in consumption of medications and treatment of diabetes, having periodontal treatment during at least the past 6 months, receiving immunosuppressive drugs, or any dietary supplements including antioxidant supplements, synbiotic products, insulin injections and the consumption of less than $90 \%$ of the prescribed capsules. A written informed consent was obtained from patients at the beginning of the study and about the confidentiality of the study was explained to the patients. All subjects were randomly divided into intervention $(n=25)$ and control $(n=25)$ groups by a random permuted block procedure. In this study, a random permuted block procedure (block design) based on the combined analysis was used to divide all subjects to 6 groups with 4 blocks in two steps plus 2 separate subjects using two codes of $\mathrm{A}$ and $\mathrm{B}$ according to the block design (AABB, BBAA, ABAB, BABA, ABBA, BABA). In this study, the participants and investigator did not inform of the allocation of subjects in either intervention or control groups (double-blinded) and the coding plan was done by a third person (who was not informed of the research) in the health-care system.

\section{Protocol of Intervention}

Subjects in the intervention group consumed one capsule/day synbiotic supplement (500 mg) (Zist Takhmir Co., Tehran, Iran) contained 7 viable and freeze-dried strains: Lactobacillus acidophilus UBLA-34 (Strain Number - ZTLac.51) $\left(2 \times 10^{9}\right.$ CFU), L. casei (Strain Number - ZT-Lca. 106) $\left(7 \times 10^{9} \mathrm{CFU}\right)$, L. rhamnosus (Strain Number $-Z t$-Lrh. 54) $\left(1.5 \times 10^{9} \mathrm{CFU}\right)$, L. bulgaricus (Strain Number - ZT-LBU. 90) $\left(2 \times 10^{8} \mathrm{CFU}\right)$, Bifidobacterium breve (Strain Number ZT-Bbr.22) $\left(2 \times 10^{9} \mathrm{CFU}\right)$, B. longum (Strain Number $-Z T$ :
Blo.105) $\left(7 \times 10^{9} \mathrm{CFU}\right)$, Streptococcus thermophilus (Strain Number - ZT-Sth.20) $\left(1.5 \times 10^{9} \mathrm{CFU}\right)$, and $100 \mathrm{mg}$ fructooligosaccharide for 8 weeks. ${ }^{30}$ Also, the subjects in the control group received one capsule/day placebo $(500 \mathrm{mg})$ in the similar packing like synbiotic supplements containing the same substance without bacteria and fructo-oligosaccharide prebiotic for 8 weeks as well. Supplement and placebo capsules were similar in terms of shape, color, taste, and size.

To ensure consuming capsules or placebo by the patients, they were reminded through phone calls or text messages and were asked to return any untaken capsules. The patients who did not consume more than $10 \%$ of the capsules were excluded from the study. Along with synbiotic supplementation during the intervention period, both groups (intervention and control) received one visit of NSPT including root surface debridement and scaling (a careful cleaning of the root surfaces to remove plaque and calculus from deep periodontal pockets and to smooth the tooth root to remove bacterial toxins). The second NSPT was performed one-month post-intervention. The patients were instructed to avoid consuming any other synbiotic products and mouthwash, maintain their usual dietary habits, lifestyle, and physical activity during the study and avoid any changes in using medication, if possible.

\section{Outcomes}

In the present study, the primary outcome was the PD and the secondary outcomes were other periodontal indices and oxidative stress biomarkers.

\section{Assessment of Demographic Data, Anthropometric Indices, and Food Intake}

Anthropometric indices and 3-day 24-h food recall were evaluated by a trained interviewer (nutritionist) at the beginning and at the end of the study. Body weight was measured in an overnight fasting status, without shoes and in minimal clothing, using an analog scale (Seca, Germany) with $0.1 \mathrm{~kg}$ accuracy. The height was measured using a stadiometer (Seca) with $0.5-\mathrm{cm}$ accuracy without shoes. BMI was calculated as the weight in kilograms divided by the height in meters squared. Waist (the widest area between the edge of the lower rib and iliac corset) and hip circumferences were measured using a tape measure with an accuracy of $0.5 \mathrm{~cm}$ at baseline and post-intervention.

\section{Blood Collection and Biochemical Assays}

A venous blood sample $(5 \mathrm{~mL})$ was collected from subjects after an overnight fasting at baseline and end of the study and 
processed for biochemical analysis. Serum markers of oxidative stress such as total antioxidant capacity (TAC), superoxide dismutase (SOD), catalase (CAT), glutathione peroxidase (GPx), and malondialdehyde (MDA) were measured by reliable spectrophotometric methods using Zell Bio GmbH kit (Germany). We assessed serum IL-1 $\beta$ by commercial cytokine-specific enzyme-linked immunosorbent assay (ELISA) kit (Human IL-1 $\beta$ Elisa kit [IBL, Germany]).

\section{Evaluation of Periodontal Status}

To evaluate the periodontal status indices including pocket depth (PD), bleeding during probe (BOP), plaque, and clinical attachment level (CAL) were assessed by a periodontologist (in order to increase accuracy) at the beginning and end of the study and recorded in the patient's dental records. Also, the calibration of various instruments such as autoclave and X-ray was done at the beginning of the study and then according to instructions of devices at regular periods. X-ray (radiographic photos) was taken from patients' mouths for further diagnosis. The PD was assessed using a UNC-15 (University of North Carolina No. 15) manual periodontal probe at 6 sites per tooth: mesiobuccal, midbuccal, distobuccal, mesiolingual, and midlingual, and distolingual sites. The selected sites with the probing depth $\geq 4 \mathrm{~mm}$ were randomly selected from at least 3 of the 4 quadrants (the term that dentists use it for the division of mouth and teeth, the mouth has 4 quadrants: upper right, upper left, lower right, and lower left) for clinical measurements after the intervention. The CAL was evaluated by a full-mouth periodontal examination and determined by measuring the distance from the cement - enamel junction to the bottom of the gingival crevice. Periodontitis is defined as severe in individuals with $\mathrm{CAL} \geq 5 \mathrm{~mm}$ (not on the same tooth) or moderate in individuals with CAL of 3-4 mm (not on the same tooth) or weak in individuals with CAL of 1-2 $\mathrm{mm}$ (not on the same tooth). BOP: Bleeding in 30 to $60 \mathrm{~s}$ after the probe is considered as 0 if no bleeding and 1 if there is bleeding. Plaque index, the amount of plaque on the tooth surface. Also, some instructions of dental hygiene such as use dental floss correctly and how to brush were trained to patients.

\section{Statistical Analysis}

All statistical analyses were performed using SPSS (version 17; SPSS Inc., Chicago, IL). All results were expressed as means \pm standard deviations (SD) for quantitative variables or number (percentage) for qualitative variables. To ensure a normal distribution of variables, the Kolmogorov-Smirnoff test was used. The Chi-square test was used to compare qualitative variables. P-value is reported based on the McNemar-test to identify within-group differences (before and after the intervention) for qualitative variables. The Paired-sample test was used to identify within-group differences (before and after the intervention) for quantitative variables. We used the Independent sample $t$-test to compare the results between two groups (placebo and synbiotic supplement). Also, the Independent sample $t$-test was used to identify changes between two groups at the end of the study. The Analysis of covariance (ANCOVA) was used to identify any differences between two groups at the end of study after adjusting for covariates. Results with $\mathrm{P}<0.05$ were considered statistically significant.

\section{Results}

\section{General Characteristics, Anthropometric Indices, and Dietary Intake}

All data in this study were normal. Forty-seven patients were recruited in this study (control group $n=24$, and intervention group $\mathrm{n}=23$ ) (Figure 1). The mean age of the subjects in the intervention and control group was $48.6 \pm$ 5.8 and $50.1 \pm 3.6$ years old, respectively. No serious adverse effects or symptoms were reported during the study related to consumption of multispecies synbiotic supplements. There were no significant differences observed in demographic characteristics include age, sex, weight, BMI, hip circumferences, waist circumferences, duration of diabetes, and medications (data not shown) between two groups at baseline $(\mathrm{p} \geq 0.05)$ (Table 1).

No significant differences were observed in dietary intakes such as energy, macronutrients, and micronutrients including antioxidant vitamins $\mathrm{C}, \mathrm{E}, \mathrm{A}$, beta-carotene, $\alpha$ tocopherol, and selenium at baseline and after the intervention in any of two groups $(\mathrm{p} \geq 0.05)$ (Table 2$)$.

\section{Periodontal Status (PD, CAL, BOP, and Plaque)}

The results of this study showed that no significant difference was observed between two groups in the mean levels of PD, CAL, BOP, and plaque at baseline. The mean PD was significantly decreased in the intervention group $(4.04 \pm 1.04 \mathrm{vs}$ $3.47 \pm 0.79 \mathrm{~mm} ; \mathrm{p}=0.042$ ) compared with control group postintervention. In addition, PD was significantly decreased in the intervention $(4.30 \pm 0.76$ vs $3.47 \pm 0.79 \mathrm{~mm} ; \mathrm{p}<0.001)$ and the control $(4.50 \pm 0.97$ vs $4.04 \pm 1.04 \mathrm{~mm} ; \mathrm{p}=0.008)$ 


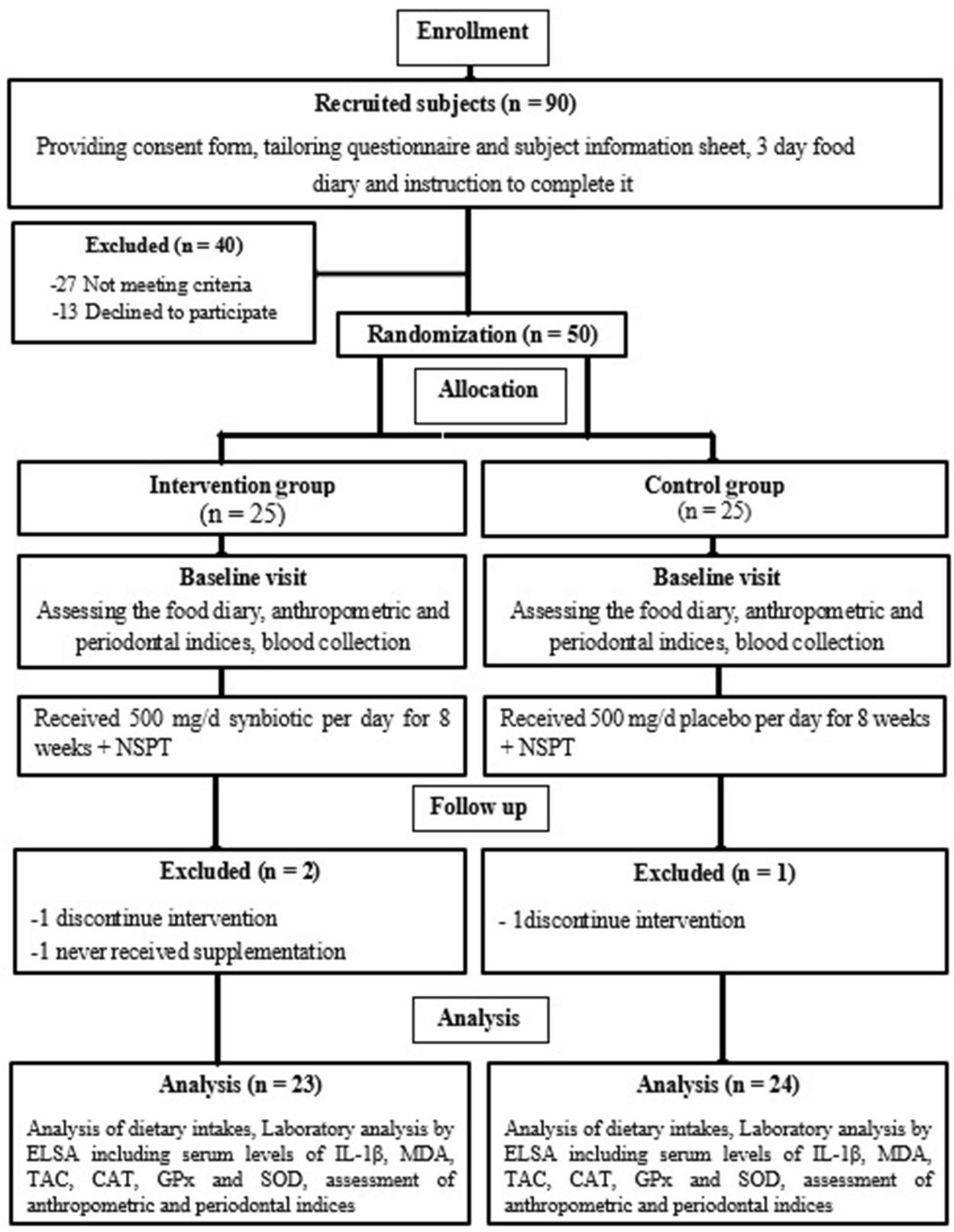

Figure I Flow diagram of the study.

Abbreviations: NSPT, non-surgical periodontal therapy; ELISA, enzyme-linked immunosorbent assay; IL-I $\beta$, interleukin-I $\beta$; MDA, malondialdehyde; TAC, total antioxidant capacity; SOD, superoxide dismutase; CAT, catalase; GPx, glutathione peroxidase.

groups after 8 weeks compared with the baseline. The mean $\mathrm{CAL}$ was also significantly reduced in the intervention group post-intervention $(3.26 \pm 0.61$ and $2.73 \pm 0.75 \mathrm{~mm} ; \mathrm{p}=0.002)$, but, in the control group tended to be significant $(3.08 \pm 0.77$ vs $2.95 \pm 0.75 \mathrm{~mm} ; \mathrm{p}=0.08)$. At the end of the study, there was a significant difference in the mean changes of CAL between the intervention and control groups $(p=0.02)$. Also, after adjusting for confounding factors, the results did not change in terms of significance. The BOP was reduced in both control and intervention groups after the intervention compared with the baseline, but none were statistically significant ( $p=0.32$ vs $p=0.07$, respectively). However, after the intervention, the reduction of BOP in the supplement group was significantly greater than the control group $(p=0.04)$. The results of McNemar-test showed plaque index significantly decreased in the intervention group compared with the baseline $(p=0.02)$, but, in the control group tended to be significant $(\mathrm{p}=0.058)$. Also, supplementation with synbiotic with NSPT resulted in a significant reduction in plaque index compared with the control group $(\mathrm{p}=0.035)$ (Table 3$)$. 
Table I Baseline Characteristics of the Subjects

\begin{tabular}{|c|c|c|c|}
\hline Variables & $\begin{array}{l}\text { Control Group } \\
(n=24)\end{array}$ & $\begin{array}{l}\text { Intervention } \\
\text { Group }(n=23)\end{array}$ & *P-value \\
\hline Age $(y)$ & $50.1 \pm 3.6$ & $48.6 \pm 5.8$ & 0.28 \\
\hline \multicolumn{4}{|l|}{ Gender } \\
\hline Female $(\mathrm{N})$ & 16 & 17 & $0.59^{\mathrm{a}}$ \\
\hline Male $(\mathrm{N})$ & 8 & 6 & \\
\hline Weight (kg) & $69 \pm 6.1$ & $68 \pm 8.9$ & 0.96 \\
\hline BMI $(\mathrm{kg} / \mathrm{m} 2)$ & $25.5 \pm 2.7$ & $24 \pm 3.6$ & 0.12 \\
\hline$W C(\mathrm{~cm})$ & $103.3 \pm 7.9$ & $106.9 \pm 6.7$ & 0.10 \\
\hline $\mathrm{HC}(\mathrm{cm})$ & $107.8 \pm 8.2$ & $108.4 \pm 8.1$ & 0.81 \\
\hline $\begin{array}{l}\text { Physical Activity } \\
\text { (met-min/week) }\end{array}$ & $351.85 \pm 34.39$ & $334.47 \pm 40.23$ & 0.77 \\
\hline $\begin{array}{l}\text { Disease duration } \\
\text { (years) }\end{array}$ & $6.66 \pm 1.57$ & $7.26 \pm 2.17$ & 0.28 \\
\hline
\end{tabular}

Notes: The results are described as mean \pm SD for quantitative data and number for qualitative data. $* P<0.05$ was considered as significant using Independent $t$-test between the two groups at baseline. ${ }^{a}<<0.05$ was considered as significant using Chi-square test.

Abbreviations: BMI, body mass index, WC, waist circumference. HC, hip circumference.

\section{Interleukin I Beta (IL-I $\beta$ ) and Malondialdehyde (MDA)}

There was no significant difference $(p \geq 0.05)$ in the mean levels of IL-1 $\beta$ and MDA between the two groups at baseline. At the end of the study, synbiotic supplementation significantly reduced serum levels of IL-1 $\beta(2.64 \pm$ 0.41 vs $2.19 \pm 0.48 \mathrm{pg} / \mathrm{mL}$, respectively; $\mathrm{p}<0.001)$ and MDA (18.25 \pm 1.89 vs $17.22 \pm 1.82 \mu \mathrm{M}$, respectively; $\mathrm{p}<$ 0.001 ) in the intervention group. Also, the mean changes of IL-1 $\beta$ and MDA were significantly lower in the intervention group compared with the control group $(\mathrm{p}=0.02$ and $\mathrm{p}<0.001$, respectively). Also, after adjusting for confounding factors, the results did not change in terms of significance (Table 4).

\section{Superoxide Dismutase (SOD), Catalase (CAT), Glutathione Peroxidase (GPx), and Total Antioxidant Capacity (TAC)}

Moreover, there was no significant difference $(p \geq 0.05)$ in the mean serum levels of SOD, CAT, GPx, and TAC between two groups at baseline. The results of this study showed that the mean serum levels of SOD, GPx, and TAC were significantly increased in the intervention group after the intervention compared with the baseline $(14.32 \pm 2.52$ vs $16.08 \pm 4.02 \mathrm{U} / \mathrm{mL}$, respectively; $p=0.003)(215.77 \pm 62.57$ vs $230.5 \pm 57.93 \mathrm{U} /$ $\mathrm{mL}$, respectively; $\mathrm{p}=0.01)$ and $(0.588 \pm 0.08$ vs $0.636 \pm$ $0.09 \mathrm{mM}$, respectively; $\mathrm{p}=0.002$ ). Also, the mean changes
Table 2 Dietary Intakes of Participants at Baseline and After the Intervention

\begin{tabular}{|c|c|c|c|}
\hline Variables & $\begin{array}{l}\text { Control Group } \\
(n=24)\end{array}$ & $\begin{array}{l}\text { Intervention } \\
\text { Group } \\
(n=23)\end{array}$ & $P$-value** \\
\hline \multicolumn{4}{|l|}{ Energy (kcal) } \\
\hline Baseline & $1962.18 \pm 318.32$ & $1946.21 \pm 345.32$ & 0.87 \\
\hline After intervention & $1985.20 \pm 313.33$ & $1959.45 \pm 312.17$ & 0.77 \\
\hline$P$-value* & 0.5 & 0.77 & \\
\hline \multicolumn{4}{|l|}{ Carbohydrate (g/d) } \\
\hline Baseline & $273.28 \pm 43.44$ & $270.73 \pm 46.18$ & 0.84 \\
\hline After intervention & $277.92 \pm 43.86$ & $267.01 \pm 46.66$ & 0.41 \\
\hline$P$-value* & 0.35 & 0.53 & \\
\hline \multicolumn{4}{|l|}{ Protein (g/d) } \\
\hline Baseline & $62.62 \pm 9.31$ & $63.42 \pm 8.92$ & 0.76 \\
\hline After intervention & $64.18 \pm 9.73$ & $61.78 \pm 13.18$ & 0.47 \\
\hline$P$-value* & 0.20 & 0.32 & \\
\hline \multicolumn{4}{|l|}{ Fat $(g / d)$} \\
\hline Baseline & $67.58 \pm 10.96$ & $67.03 \pm 11.89$ & 0.87 \\
\hline After intervention & $68.37 \pm 10.79$ & $66.44 \pm 12.53$ & 0.57 \\
\hline$P$-value* & 0.50 & 0.74 & \\
\hline \multicolumn{4}{|l|}{ Saturated fat $(g / d)$} \\
\hline Baseline & $22.90 \pm 7.35$ & $26.83 \pm 9.57$ & 0.12 \\
\hline After intervention & $24.35 \pm 10.38$ & $23.25 \pm 9.46$ & 0.70 \\
\hline$P$-value* & 0.20 & 0.59 & \\
\hline \multicolumn{4}{|l|}{ Cholesterol (mg/d) } \\
\hline Baseline & $197.66 \pm 59.23$ & $173.99 \pm 48.75$ & 0.31 \\
\hline After intervention & $171.60 \pm 43.60$ & $158.98 \pm 31.55$ & 0.82 \\
\hline$P$-value* & 0.90 & 0.18 & \\
\hline \multicolumn{4}{|l|}{ Selenium $(\mu g / d)$} \\
\hline Baseline & $60.21 \pm 15.11$ & $61.55 \pm 15.20$ & 0.76 \\
\hline After intervention & $64.62 \pm 15.91$ & $68.43 \pm 12.62$ & 0.36 \\
\hline$P$-value* & 0.30 & 0.13 & \\
\hline \multicolumn{4}{|l|}{ Vitamin A $(\mu \mathrm{g} / \mathrm{d})$} \\
\hline Baseline & $455 \pm 164.10$ & $448.24 \pm 209.48$ & 0.89 \\
\hline After intervention & $503 \pm 147.32$ & $532.74 \pm 132.39$ & 0.48 \\
\hline$P$-value* & 0.20 & 0.16 & \\
\hline \multicolumn{4}{|l|}{ Vitamin C $(\mathrm{mg} / \mathrm{d})$} \\
\hline Baseline & $84.58 \pm 26.85$ & $93.82 \pm 23.01$ & 0.21 \\
\hline After intervention & $86.76 \pm 28.83$ & $89.49 \pm 28.71$ & 0.74 \\
\hline$P$-value* & 0.80 & 0.52 & \\
\hline \multicolumn{4}{|l|}{ Vitamin E (mg/d) } \\
\hline Baseline & $12.74 \pm 1.96$ & $12.10 \pm 1.82$ & 0.25 \\
\hline After intervention & $12.10 \pm 3.46$ & $\mid 3.14 \pm 2.51$ & 0.24 \\
\hline$P$-value* & 0.48 & 0.13 & \\
\hline
\end{tabular}

Notes: The results are described as mean \pm SD. $* *$ Difference between groups at baseline and post-intervention (Independent $t$-test). *Within-group difference (Paired $t$-test). $P<0.05$ was considered significant.

of SOD and GPx were significantly higher in the intervention group compared with the control group $(p=0.007$ and $p=0.01$, respectively). However, no significant changes were seen in 
Table 3 Periodontal Status at Baseline and Post-Intervention

\begin{tabular}{|c|c|c|c|c|c|}
\hline Variables & Control Group $(n=24)$ & Intervention Group $(n=23)$ & $P$-value & *P-value & **P-value \\
\hline \multicolumn{6}{|l|}{ PD (mm) } \\
\hline Baseline & $4.50 \pm 0.97$ & $4.30 \pm 0.76$ & $0.45^{\mathrm{a}}$ & & \\
\hline After intervention & $4.04 \pm 1.04$ & $3.47 \pm 0.79$ & $0.04^{\mathrm{b}}$ & & \\
\hline$P$-value ${ }^{c}$ & 0.008 & $<0.001$ & & & \\
\hline Difference & $-0.45 \pm 0.77$ & $-0.82 \pm 0.93$ & & 0.15 & 0.59 \\
\hline \multicolumn{6}{|l|}{$\mathrm{CAL}(\mathrm{mm})$} \\
\hline Baseline & $3.08 \pm 0.77$ & $3.26 \pm 0.61$ & $0.39^{\mathrm{a}}$ & & \\
\hline After intervention & $2.95 \pm 0.75$ & $2.73 \pm 0.75$ & $0.32^{\mathrm{b}}$ & & \\
\hline$P$-value ${ }^{c}$ & 0.083 & 0.002 & & & \\
\hline Difference & $-0.12 \pm 0.33$ & $-0.52 \pm 0.73$ & & 0.02 & 0.016 \\
\hline \multicolumn{6}{|l|}{$\mathrm{BOP}(+)$} \\
\hline Baseline $(\mathrm{N})$ & 24 & 23 & $0.35^{\mathrm{d}}$ & & \\
\hline After intervention $(\mathrm{N})$ & 22 & 19 & $0.048^{d}$ & & \\
\hline$P$-value ${ }^{e}$ & 0.32 & 0.07 & & & \\
\hline \multicolumn{6}{|l|}{ Plaque $(+)$} \\
\hline Baseline $(N)$ & 24 & 23 & $0.17^{\mathrm{d}}$ & & \\
\hline After intervention $(\mathrm{N})$ & 19 & 14 & $0.035^{d}$ & & \\
\hline$P$-value ${ }^{e}$ & 0.058 & 0.024 & & & \\
\hline
\end{tabular}

Notes: Values are expressed as means \pm SD. $P<0.05$ was considered as significant. ${ }^{\text {a }}<0.05$ was considered as significant using Independent $t$-test between the two groups at baseline. ${ }^{b} \mathrm{P}<0.05$ was considered as significant using Independent $t$-test between the two groups post-intervention. ${ }^{c} W$ ithin-group difference, $p$-value is reported based on Paired $t$-test ${ }^{d}$ Difference between groups at baseline and post intervention. $P<0.05$ was considered as significant using Chi-square test. ${ }^{e} W$ ithin-group difference, $p$-value is reported based on McNemar-test $* \mathrm{P}<0.05$ was considered as significant difference using Independent $t$-test between the two groups post-intervention $* * P<0.05$ was considered as significant difference using Analysis of covariance (ANCOVA) between the two groups post-intervention after adjusting for confounding factors.

Abbreviations: PD, pocket depth; CAL, clinical attachment loss.

the mean serum levels of CAT after the intervention. Also, after adjusting for confounding factors, the results did not change in terms of significance (Table 4).

\section{Discussion}

To the best of authors' knowledge, there is no study evaluates the effects of synbiotic supplementation in adjunct with NSPT on periodontal status, inflammatory, and antioxidant parameters in T2DM patients with CP. The results of this study showed that synbiotic supplementation with NSPT significantly decreased the mean levels of IL-1 $\beta$, MDA, $\mathrm{CAL}$, plaque index, and $\mathrm{PD}$ in the intervention group. Moreover, serum levels of TAC, SOD, and GPx were significantly increased in the intervention group. Also, there were significant differences in the mean changes of IL-1 $\beta$, MDA, TAC, GPx, BOP, plaque index, and CAL between the intervention and control groups after the intervention.

Using antibiotics in excess amounts to treat infections can result in the growth of resistant microbial strains, which may increase the risk of pathogen overgrowth in patients. ${ }^{31}$ Therefore, there is an urgent need for alternative therapeutic approaches to treat infections. So, the necessity of using prebiotics and/or probiotics as therapeutic health approaches would be inevitable. Traditionally, pre- and probiotics have been used to provide health benefits for the gastrointestinal tract. However, a huge interest in research has been devoted to use either (or both) pre- and probiotic for non-gut applications such as oral health products to protect against dental caries. ${ }^{32}$ The emphasis in using pre- and probiotics in oral health applications is mainly to control cariogenic streptococci (colonizing in the mouth) and diseases such as periodontitis, dental caries, halitosis, and candidiasis. ${ }^{33}$

\section{Synbiotic and Periodontal Indices}

There is no human study investigating the effects of synbiotic along with NSPT on periodontal indices in T2DM with CP. There is a two-way relationship between periodontal disease and several systemic pathologies such as DM. It is conceived that controlling one of these two pathologies may be beneficial in controlling the other. ${ }^{34}$ The results of the present study showed that consumption of synbiotic for 8 weeks along with NSPT significantly decreased the mean of PD, and CAL after the intervention. Also, number of patients with plaque and the number of them that suffered from 
Table 4 The Mean \pm SD of Antioxidant and Inflammatory Markers at Baseline and Post-Intervention

\begin{tabular}{|c|c|c|c|c|c|}
\hline Variables & Intervention Group $(n=23)$ & Control Group $(n=24)$ & $P$-value ** & $P$-value**** & $P$-value e**** $^{*}$ \\
\hline $\begin{array}{l}\text { IL-Ib (pg/mL) } \\
\text { Baseline } \\
\text { After } 8 \text { weeks } \\
\text { P-value * } \\
\text { Difference }\end{array}$ & $\begin{array}{l}2.64 \pm 0.41 \\
2.19 \pm 0.48 \\
<0.001 \\
-0.45 \pm 0.42\end{array}$ & $\begin{array}{l}2.75 \pm 0.45 \\
2.61 \pm 0.65 \\
0.18 \\
-0.13 \pm 0.47\end{array}$ & $\begin{array}{l}0.42 \\
0.02\end{array}$ & 0.02 & 0.045 \\
\hline $\begin{array}{l}\text { MDA }(\mu \mathrm{M}) \\
\text { Baseline } \\
\text { After } 8 \text { weeks } \\
\text { P-value* } \\
\text { Difference }\end{array}$ & $\begin{array}{l}18.25 \pm 1.89 \\
17.22 \pm 1.82 \\
<0.001 \\
-1.02 \pm 0.95\end{array}$ & $\begin{array}{l}18.53 \pm 1.37 \\
18.4 \pm 1.33 \\
0.11 \\
-0.13 \pm 0.39\end{array}$ & $\begin{array}{l}0.57 \\
0.01\end{array}$ & . & 0.003 \\
\hline $\begin{array}{l}\text { TAC (mM) } \\
\text { Baseline } \\
\text { After } 8 \text { weeks } \\
\text { P-value* } \\
\text { Difference }\end{array}$ & $\begin{array}{l}0.588 \pm 0.08 \\
0.636 \pm 0.09 \\
0.002 \\
0.04 \pm 0.06\end{array}$ & $\begin{array}{l}0.636 \pm 0.131 \\
0.655 \pm 0.179 \\
0.48 \\
0.01 \pm 0.12\end{array}$ & $\begin{array}{l}0.15 \\
0.66\end{array}$ & 0.35 & 0.88 \\
\hline $\begin{array}{l}\text { SOD }(\mathrm{U} / \mathrm{mL}) \\
\text { Baseline } \\
\text { After } 8 \text { weeks } \\
\text { P-value* } \\
\text { Difference }\end{array}$ & $\begin{array}{l}14.32 \pm 2.52 \\
16.08 \pm 4.02 \\
0.003 \\
1.75 \pm 2.49\end{array}$ & $\begin{array}{l}14.82 \pm 2.36 \\
14.99 \pm 2.34 \\
0.12 \\
0.16 \pm 0.48\end{array}$ & $\begin{array}{l}0.5 \\
0.27\end{array}$ & 0.007 & 0.01 \\
\hline $\begin{array}{l}\text { CAT }(\mathrm{U} / \mathrm{mL}) \\
\text { Baseline } \\
\text { After } 8 \text { weeks } \\
\text { P-value* } \\
\text { Difference }\end{array}$ & $\begin{array}{l}24.5 \pm 4.36 \\
25.1 \pm 4.14 \\
0.11 \\
0.44 \pm 5.33\end{array}$ & $\begin{array}{l}23.59 \pm 3.37 \\
24.04 \pm 4.93 \\
0.69 \\
0.44 \pm 5.33\end{array}$ & $\begin{array}{l}0.44 \\
0.44\end{array}$ & 0.90 & 0.50 \\
\hline $\begin{array}{l}\text { GPX }(\mathrm{U} / \mathrm{mL}) \\
\text { Baseline } \\
\text { After } 8 \text { weeks } \\
\text { P-value* } \\
\text { Difference }\end{array}$ & $\begin{array}{l}215.77 \pm 62.57 \\
230.5 \pm 57.93 \\
0.01 \\
14.72 \pm 24.9\end{array}$ & $\begin{array}{l}218.27 \pm 66.87 \\
215 \pm 61.97 \\
0.5 \\
-3.27 \pm 22.86\end{array}$ & $\begin{array}{l}0.89 \\
0.39\end{array}$ & 0.01 & 0.03 \\
\hline
\end{tabular}

Notes: Values are expressed as means \pm SD. $* P<0.05$ was considered as significant using Paired $t$-test. $* * P<0.05$ was considered as significant using Independent $t$-test between the two groups at baseline and post-intervention. $* * * P<0.05$ was considered as significant difference using Independent $t$-test between the two groups postintervention. ${ }^{* * * *} P<0.05$ was considered as significant difference using Analysis of covariance (ANCOVA) between the two groups post-intervention after adjusting for confounding factors.

Abbreviations: IL-I $\beta$, Interleukin-I $\beta$; MDA, malondialdehyde; TAC, total antioxidant capacity; SOD, superoxide dismutase; CAT, catalase; GPx, glutathione peroxidase.

BOP were decreased in the intervention group compared to the control group. Similarly, Vivekananda et al showed that Lactobacilli reuteri prodentis lozenges alone or in along with scaling and root planing (SRP) decreased pocket depth (PD), clinical attachment level (CAL), and also reduced microbiological levels of the pathogens Aggregibacter actinomycetemcomitans (Aa), Porphyromonas gingivalis (Pg), and Prevotella intermedia (Pi) in systemically healthy chronic periodontitis patients. ${ }^{14}$ Also, another study found that the administration of Lactobacillus in the form of tablets/ lozenges reduced gingival inflammation and decreased the probing pocket depth. ${ }^{35}$ Moreover, in agreement with the findings of the present study, NSPT significantly improved all periodontal parameters in patients with T2DM and chronic periodontitis after 3 months. ${ }^{19}$ However, in this study, only PD was significantly decreased in the control group. NSPT without using any supplement may be considered important.

The most common pathogens causing periodontal disease are including Tannarella forsythia $i$ Aggregatibacter actinomycetemcomitans, Porphyromonas gingivalis, and Treponema denticola. ${ }^{36}$ Actually, lipopolysaccharide is the main component of the outer membrane of Gram-negative bacteria, which can be increased in plaque during the 
development of gingivitis and stimulates an inflammatory response in host cells. ${ }^{37}$ The mechanism by which these strains can inhibit the LPS-stimulated release of inflammatory mediators from host cells has not been revealed and warrants investigation in future studies. However, previous research showed that probiotic strains used in the gut can interact with toll-like receptors on host cells to downregulate the expression of nuclear factor-kappa-B and proinflammatory cytokines. ${ }^{38}$ Probiotics release two bacteriocins (reuterin and reutericiklin) which change the way the immune system reacts or they prevent the distribution of pathogens. It has been shown that using probiotics can reduce bleeding gingiva and gingivitis. ${ }^{39}$ Short peptides produced by Lactobacillus helveticus stimulate osteoblasts to grow bones, so that it has his role in reducing bones' resorption connected with periodontitis. ${ }^{40}$

\section{The Effects of Synbiotic on Inflammatory and Antioxidant Parameters}

The findings of this study indicated that the consumption of synbiotic supplement along with NSPT for 8 weeks significantly decreased the mean serum levels of IL-1 $\beta$ and MDA in the intervention group. Also, serum levels of TAC, SOD, and GPx were significantly increased in the intervention group. Similar to the present study, in a double-blinded clinical trial, diabetic patients received $300 \mathrm{~g}$ /day probiotic yogurt fermented with $7 \times 10^{6} \mathrm{CFU}$ Bifidobacterium lactis Bb12 and $6 \times 10^{6}$ CFU L and acidophilus La5 for 6 weeks. The results showed that probiotics significantly improved glycaemic control and lipid profiles, increased oxidative stress parameters such as SOD, GPx, and TAC and decreased MDA. ${ }^{17}$ Similar to this study, Bahmani et al reported that the consumption of synbiotic bread containing viable and the heat-resistant probiotic Lactobacillus sporogenes $\left(1 \times 10^{8} \mathrm{CFU}\right)$ and $0.07 \mathrm{~g}$ inulin per $1 \mathrm{~g}$ for 8 weeks significantly increased plasma $\mathrm{NO}$ and reduced MDA in compare to the probiotic bread containing Lactobacillus sporogenes $\left(1 \times 10^{8} \mathrm{CFU}\right)$ and control bread, but contrary to this study, not find any significant effect of the synbiotic bread consumption on plasma TAC, and plasma GSH in compare to the probiotic and control breads. Also, the CAT did not change significantly in both studies. ${ }^{41}$ The differences in the type of probiotic bacteria, dosage of bacterial strain, and the type of prebiotic may be reasons behind obtaining different results. Similarly, in another meta-analysis study (2018) it was shown that supplementation with synbiotic or probiotic significantly increased levels of oxidative stress parameters including TAC, GSH, SOD, and NO. Moreover, the levels of MDA were significantly decreased. ${ }^{42}$ It is suggested that synbiotics can improve the composition of gut microflora and reduce oxidative stress by changing the composition of microflora and reducing LPS concentration. Recently, it has been shown that intestinal bacteria (probiotics) can reduce the production of $\mathrm{NF}-\kappa \mathrm{B}$, which can interfere with the transcription of a large number of inflammatory genes and decrease oxidative stress. ${ }^{43}$ One of the limitations of this study was the selection of only two groups (due to financial constraints and some executive problems such as the low number of cases). Therefore, it is recommended to do other studies with 4 study groups in future (including group1; Diabetes + no periodontal treatment + placebo, group2; Diabetes + no periodontal treatment + synbiotic, group3; Diabetes + NSPT + placebo, group4; Diabetes + NSPT + synbiotic). Also, we did not measure the levels of blood glucose and lipid profile, which was another limitation of our study. In conclusion, it is suggested that supplementation with synbiotic in adjunct with NSPT in T2DM patients with CP may have beneficial effects on serum levels of some inflammatory markers, oxidative status, and periodontal parameters.

\section{Abbreviations}

DM, diabetes mellitus; $\mathrm{CP}$, chronic periodontitis; TNF- $\alpha$, tumor necrosis factor-alpha; IL-6, interleukin-6; Hs-CRP, hs-C-reactive protein; IL-1 $\beta$, interleukin-1 $\beta$; MDA, malondialdehyde; TAC, total antioxidant capacity; SOD, superoxide dismutase; CAT, catalase; GPx, glutathione peroxidase; CAL, clinical attachment loss; BOP, bleeding on probing; PD, pocket depth; NSPT, non-surgical periodontal therapy; WC, waist circumference; HC, hip circumference; WHR, waist to hip ratio; ELISA, enzyme-linked immunosorbent assay.

\section{Compliance with Ethical Standards}

In the present study, written informed consent was obtained from all patients before initiating the study. Also, study design was done according to the guidelines of the Helsinki Declaration and all procedures involving human patients were approved by the Ethics Committee of Ahvaz Jundishapur University of Medical Sciences (Ethical Code. IR.AJUMS.REC.1396.578).

\section{Data Sharing Statement}

The datasets are not publicly available because of lack of agreement for disclosing individual raw data in public but are available from the corresponding author on reasonable request. 


\section{Acknowledgments}

Authors express thanks for the Nutrition and Metabolic Disorders Research Center, and Research Center for Diabetes, Endocrinology and Metabolism clinic employees of Golestan Hospital and Dental Clinic of Ahvaz Jundishapur University of Medical Sciences.

\section{Funding}

This research project was sponsored by the ViceChancellor for Research Affairs of Ahvaz Jundishapur University of Medical Sciences (NRC-9612).

\section{Disclosure}

The authors have declared that there are no conflicts of interest in this work.

\section{References}

1. Preshaw PM, de Silva N, McCracken GI, et al. Compromised periodontal status in an urban Sri Lankan population with type 2 diabetes. J Clin Periodontol. 2010;37(2):165-171. doi:10.1111/cpe.2010.37. issue-2

2. Navarro-Sanchez AB, Faria-Almeida R, Bascones-Martinez A. Effect of non-surgical periodontal therapy on clinical and immunological response and glycaemic control in type 2 diabetic patients with moderate periodontitis. J Clin Periodontol. 2007;34(10):835-843. doi:10.1111/cpe.2007.34.issue-10

3. Genco RJ, Grossi SG, Ho A, Nishimura F, Murayama Y. A proposed model linking inflammation to obesity, diabetes, and periodontal infections. $J$ Periodontol. 2005;76(11 Suppl):2075-2084. doi:10.1902/jop.2005.76.11-S.2075

4. Weidlich P, Cimões R, Pannuti CM, Oppermann RV. Association between periodontal diseases and systemic diseases. Braz Oral Res. 2008;22:32-43. doi:10.1590/S1806-83242008000500006

5. Allen E, Matthews J, O'Connor R, O'Halloran D, Chapple I. Periodontitis and type 2 diabetes: is oxidative stress the mechanistic link? Scott Med J. 2009;54(2):41-47. doi:10.1258/rsmsmj.54.2.41

6. Akalin FA, Baltacioglu E, Alver A, Karabulut E. Lipid peroxidation levels and total oxidant status in serum, saliva and gingival crevicular fluid in patients with chronic periodontitis. J Clin Periodontol. 2007;34(7):558-565. doi:10.1111/j.1600-051X.2007.01091.x

7. Ganjifrockwala FA, Joseph JT, George G. Decreased total antioxidant levels and increased oxidative stress in South African type 2 diabetes mellitus patients. J Endocrinol Metabol Diabetes South Africa. 2017;22(2):21-25. doi:10.1080/16089677.2017.1324590

8. Thomas B, Rao A, Prasad BR, Kumari S. Serum levels of antioxidants and superoxide dismutase in periodontitis patients with diabetes type 2. J Indian Soc Periodontol. 2014;18(4):451. doi:10.4103/ 0972-124X.138686

9. Grossi SG, Skrepcinski FB, DeCaro T, et al. Treatment of periodontal disease in diabetics reduces glycated hemoglobin. $J$ Periodontol. 1997;68(8):713-719. doi:10.1902/jop.1997.68.8.713

10. Ou L, Li R. Effect of periodontal treatment on glycosylated hemoglobin levels in elderly patients with periodontal disease and type 2 diabetes. Chin Med J. 2011;124(19):3070-3073.

11. Asemi Z, Khorrami-Rad A, Alizadeh S-A, Shakeri H, Esmaillzadeh A. Effects of synbiotic food consumption on metabolic status of diabetic patients: a double-blind randomized cross-over controlled clinical trial. Clin Nutri. 2014;33(2):198-203. doi:10.1016/j.clnu.2013.05.015
12. Chen C, Yu X, Lu H, Xiao D, Mao W, Li L. Antioxidant protective effects of lactitol against endotoxemia in patients with chronic viral hepatitis. Mol Med Rep. 2013;7(2):401-405. doi:10.3892/mmr.2012.1188

13. Lye H-S, Kuan C-Y, Ewe J-A, Fung W-Y, Liong M-T. The improvement of hypertension by probiotics: effects on cholesterol, diabetes, renin, and phytoestrogens. Int J Mol Sci. 2009;10(9):3755-3775. doi:10.3390/ijms10093755

14. Vivekananda M, Vandana K, Bhat K. Effect of the probiotic Lactobacilli reuteri (Prodentis) in the management of periodontal disease: a preliminary randomized clinical trial. J Oral Microbiol. 2010;2(1):5344. doi:10.3402/jom.v2i0.5344

15. Mallappa RH, Rokana N, Duary RK, Panwar H, Batish VK, Grover S. Management of metabolic syndrome through probiotic and prebiotic interventions. Indian J Endocrinol Metab. 2012;16 (1):20-27. doi:10.4103/2230-8210.91178

16. Kullisaar T, Songisepp E, Mikelsaar M, Zilmer K, Vihalemm T, Zilmer M. Antioxidative probiotic fermented goats' milk decreases oxidative stress-mediated atherogenicity in human subjects. $\mathrm{Br}$ J Nutri. 2003;90(2):449-456. doi:10.1079/BJN2003896

17. Ejtahed HS, Mohtadi-Nia J, Homayouni-Rad A, Niafar M, AsghariJafarabadi M, Mofid V. Probiotic yogurt improves antioxidant status in type 2 diabetic patients. Nutrition. 2012;28(5):539-543. doi:10.1016/j. nut.2011.08.013

18. Sabatini S, Lauritano D, Candotto V, Silvestre F, Nardi GM. Oral probiotics in the management of gingivitis in diabetic patients: a double blinded randomized controlled study. J Biol Regul Homeost Agents. 2017;31(2 Suppl 1):197-202.

19. Mammen J, Vadakkekuttical RJ, George JM, Kaziyarakath JA, Radhakrishnan C. Effect of non-surgical periodontal therapy on insulin resistance in patients with type II diabetes mellitus and chronic periodontitis, as assessed by C-peptide and the Homeostasis Assessment Index. J Investig Clin Dent. 2017;8(3):e12221. doi:10.1111/jicd.2017.8.issue-3

20. Lambert JM, Bongers RS, de Vos WM, Kleerebezem M. Functional analysis of four bile salt hydrolase and penicillin acylase family members in Lactobacillus plantarum WCFS1. Appl Environ Microbiol. 2008;74(15):4719-4726. doi:10.1128/AEM.00137-08

21. Yadav H, Jain S, Sinha P. Antidiabetic effect of probiotic dahi containing Lactobacillus acidophilus and Lactobacillus casei in high fructose fed rats. Nutrition. 2007;23(1):62-68. doi:10.1016/j.nut.2006.09.002

22. Kullisaar T, Zilmer M, Mikelsaar M, et al. Two antioxidative lactobacilli strains as promising probiotics. Int J Food Microbiol. 2002;72 (3):215-224. doi:10.1016/S0168-1605(01)00674-2

23. Peran L, Camuesco D, Comalada M, et al. Lactobacillus fermentum, a probiotic capable to release glutathione, prevents colonic inflammation in the TNBS model of rat colitis. Int J Colorectal Dis. 2006;21 (8):737-746. doi:10.1007/s00384-005-0773-y

24. Shadnousha M, Hosseinia RS, Mehrabib Y, et al. Probiotic yogurt affects pro- and anti-inflammatory factors in patients with inflammatory Bowel disease. Iran J Pharm Res. 2013;12(4):929-936.

25. Hegazy SK, El-Bedewy MM. Effect of probiotics on pro-inflammatory cytokines and NF-KB activation in ulcerative colitis. World J Gastroenterol. 2010;16(33):4145. doi:10.3748/wjg.v16.i33.4145

26. Christgau M, Palitzsch KD, Schmalz G, Kreiner U, Frenzel S. Healing response to non-surgical periodontal therapy in patients with diabetes mellitus: clinical, microbiological, and immunologic results. J Clin Periodontol. 1998;25(2):112-124. doi:10.1111/j.1600051X.1998.tb02417.x

27. Duarte PM, da Rocha M, Sampaio E, et al. Serum levels of cytokines in subjects with generalized chronic and aggressive periodontitis before and after non-surgical periodontal therapy: a pilot study. J Periodontol;2010;81(7):1056-1063.

28. Hosseini SA, Ghaedi E, Zakerkish M, et al. Effects of ginseng extract on chemerin, apelin and glycemic biomarkers in type 2 diabetic patients. Indian J Physiol Pharmacol;2017;61(2):152-158. 
29. Zare Javid A, Seal CJ, Heasman P, Moynihan PJ. Impact of a customised dietary intervention on antioxidant status, dietary intakes and periodontal indices in patients with adult periodontitis. J Human Nutri Dietetics. 2014;27(6):523-532. doi:10.1111/jhn.2014.27.issue-6

30. Sepideh A, Karim P, Hossein A, et al. Effects of multistrain probiotic supplementation on glycemic and inflammatory indices in patients with nonalcoholic fatty liver disease: a double-blind randomized clinical trial. J Am Coll Nutr. 2016;35(6):500-505. doi:10.1080/ 07315724.2015.1031355

31. Ventola CL. The antibiotic resistance crisis: part 1: causes and threats. Pharm Ther. 2015;40(4):277.

32. Maitra A, Rollins M, Tran L, Al-Ghazzewi F, Tester R. Prebiotic konjac glucomannan hydrolysate reduces Streptococcus mutans in oral bio films. Int Assoc Dent Res (IADR) Abstracts. 2013;20-23.

33. Tester RF, Al-Ghazzewi FH. Role of prebiotics and probiotics in oral health. Nutri Food Sci. 2018;48(1):16-29. doi:10.1108/NFS-03-20170056

34. Grover HS, Luthra S. Molecular mechanisms involved in the bidirectional relationship between diabetes mellitus and periodontal disease. $J$ Indian Soc Periodontol. 2013;17(3):292. doi:10.4103/0972-124X.115642

35. Deepa D, Mehta D. Is the role of probiotics friendly in the treatment of periodontal diseases! J Indian Soc Periodontol. 2009;13(1):30. doi:10.4103/0972-124X.51892

36. Elavarasu S, Jayapalan P, Murugan T. Bugs that debugs: probiotics. J Pharm Bioallied Sci. 2012;4(Suppl 2):S319. doi:10.4103/09757406.100286
37. Schmitter T, Fiebich BL, Fischer JT, et al. Ex vivo anti-inflammatory effects of probiotics for periodontal health. J Oral Microbiol. 2018;10 (1):1502027. doi:10.1080/20002297.2018.1502027

38. Plaza-Diaz J, Gomez-Llorente C, Fontana L, Gil A. Modulation of immunity and inflammatory gene expression in the gut, in inflammatory diseases of the gut and in the liver by probiotics. World $J$ Gastroenterol. 2014;20(42):15632. doi:10.3748/wjg.v20.i42.15632

39. Bakarcic D, Jokic NI, Hrvatin S, Mikulicic A, Culav T. The use of probiotics in dental medicine. Madridge J Dent Oral Surg. 2017;1:46-48.

40. Bonifait L, Chandad F, Grenier D. Probiotics for oral health: myth or reality? J Can Dent Assoc (Tor). 2009;75:8.

41. Bahmani F, Tajadadi-Ebrahimi M, Kolahdooz F, et al. The consumption of synbiotic bread containing Lactobacillus sporogenes and inulin affects nitric oxide and malondialdehyde in patients with type 2 diabetes mellitus: randomized, double-blind, placebo-controlled trial. J Am Coll Nutr. 2016;35(6):506-513. doi:10.1080/07315724.2015.1032443

42. Heshmati J, Farsi F, Shokri F, et al. A systematic review and meta-analysis of the probiotics and synbiotics effects on oxidative stress. J Funct Foods. 2018;46:66-84. doi:10.1016/j.jff.2018.04.049

43. Bharrhan S, Koul A, Chopra K, Rishi P. Catechin suppresses an array of signalling molecules and modulates alcohol-induced endotoxin mediated liver injury in a rat model. PLOS ONE. 2011;6(6):e20635. doi:10.1371/journal.pone.0020635

Diabetes, Metabolic Syndrome and Obesity: Targets and Therapy

Dovepress

\section{Publish your work in this journal}

Diabetes, Metabolic Syndrome and Obesity: Targets and Therapy is an international, peer-reviewed open-access journal committed to the rapid publication of the latest laboratory and clinical findings in the fields of diabetes, metabolic syndrome and obesity research. Original research, review, case reports, hypothesis formation, expert opinion and commentaries are all considered for publication. The manuscript management system is completely online and includes a very quick and fair peer-review system, which is all easy to use. Visit http://www.dovepress.com/testimonials.php to read real quotes from published authors.

Submit your manuscript here: https://www.dovepress.com/diabetes-metabolic-syndrome-and-obesity-targets-and-therapy-journal 